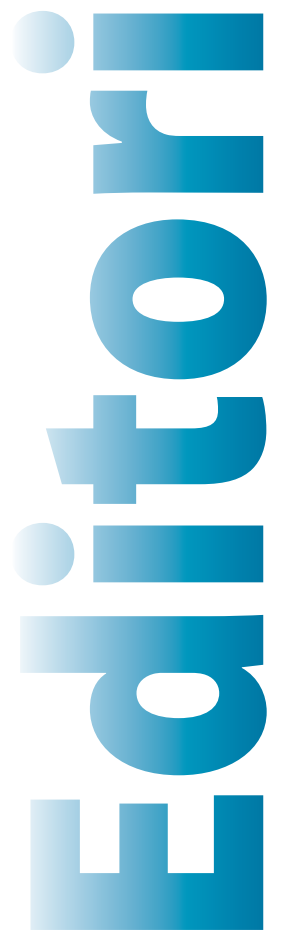

\title{
Herbes, épices et aromates
}

Les épices, de si petits ingrédients, de si grands effets, tel est le sentiment que l'on ressent après la lecture de ce dossier inédit. On comprend dès lors qu'elles aient été l'objet de tant de convoitises au long des siècles et des millénaires ainsi que nous le rappelle Eric Birlouez. On comprend moins quelles soient tombées dans l'oubli, si ce n'est que la médecine des prothèses et des médicaments, voire la diététique, ont oublié les petits et les faibles (épices, herbes, aromates...) en chemin. Mais saveur, odeurs, arômes et couleurs ont heureusement reconquis nos cuisines et nos palais à travers mille et une recettes (on évoque les mille

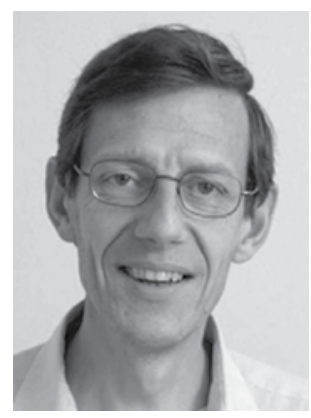
et une nuits...) ainsi que nous l'expose avec délectation Michèle Cahuzac-Picaud. Ce sont les effets antioxydants des épices qui ont d'abord retenu l'attention. Mais d'autres effets en ont fait aussi des vedettes, par exemple les effets anti-inflammatoires et les effets métaboliques du curcumin, expliquai-je, ou bien les effets antalgiques de Capsicum nous l'expose Paul Goetz; mais c'est sans compter sur des effets très particuliers, antiémétiques en l'occurrence, d'autres rhizomes tels que le gingembre nous détaille Franck Gigon ; ou encore les effets ubiquitaires et étonnants des petites merveilles de la Guadeloupe ainsi que Muriel Biabiny de l'équipe de Henri Joseph nous le montre. Le safran pourrait être un véritable allié thérapeutique ainsi que le suggère Audrey Crozet dans une monographie (dont la seconde partie est à suivre dans un prochain numéro de Phytothérapie) : ce n'est pas pour rien qu'il s'agit de l'épice la plus précieuse et la plus rare. Jean-Michel Borys donne aux épices une note éducative dans la gestion du poids : véritable assistance pédagogique, d'autant plus qu'elles sont le substitut idéal du sel nous le rappelle Pierre Meneton dans un réquisitoire implacable contre l'excès de sel. La touche délicate revient aux vertus d'une herbe banale, le persil, sous la plume légère de Daniel Wuyts.

Ce numéro une fois de plus donne envie de manger et de savoir. Mais dans la mesure où savoir vient de saveur... voici notre intellect réconcilié avec notre assiette !

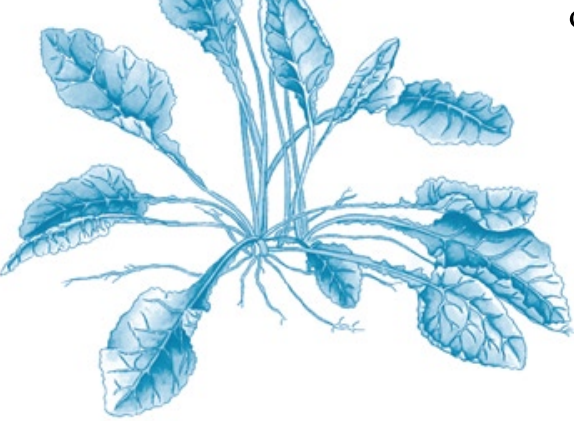

J.-M. Lecerf

Service de nutrition, institut Pasteur de Lille,

1, rue du Professeur-Calmette, BP 245, F-59019 Lille cedex, France 